Chem. Ber. 116, $3685-3691$ (1983)

\title{
Thermisch induzierte Umlagerung von (Acyloxymethyl)diorganylsilanen
}

\section{Reinhold Tacke* und Hartwig Lange}

Institut für Anorganische und Analytische Chemie der Technischen Universităt Braunschweig, Pockelsstraße 4, D-3300 Braunschweig

Eingegangen am 28. Februar 1983

Die (Acyloxymethyl)diorganylsilane $\mathrm{R}^{1} \mathrm{R}^{2} \mathrm{Si}(\mathrm{H}) \mathrm{CH}_{2} \mathrm{OC}(\mathrm{O}) \mathrm{R}^{3}(2 \mathrm{a}-\mathrm{d}$ ) unterliegen einer thermisch induzierten Umlagerung $\mathrm{zu}$ den entsprechenden Acyloxy(methyl)diorganylsilanen $\mathbf{R}^{1} \mathbf{R}^{2}$ Si$\left(\mathrm{CH}_{3}\right) \mathrm{OC}(\mathrm{O}) \mathrm{R}^{3}(3 \mathrm{a}-\mathrm{d})$. Diese Reaktion beinhaltet formal einen Austausch des am Silicium gebundenen Wasserstoffs mit dem am Kohlenstoff gebundenen Acyloxy-Rest. Bezüglich der 1,2Wasserstoff-Verschiebung konnte experimentell ein intramolekularer Prozeß bewiesen werden.

\section{Thermally Induced Rearrangement of (Acyloxymethyl)diorganylsilanes}

The (acyloxymethyl)diorganylsilanes $\mathrm{R}^{1} \mathrm{R}^{2} \mathrm{Si}(\mathrm{H}) \mathrm{CH}_{2} \mathrm{OC}(\mathrm{O}) \mathrm{R}^{3}(\mathbf{2} \mathbf{a}-\mathbf{d})$ rearrange to the corresponding acyloxy(methyl)diorganylsilanes $\mathrm{R}^{1} \mathrm{R}^{2} \mathrm{Si}\left(\mathrm{CH}_{3}\right) \mathrm{OC}(\mathrm{O}) \mathrm{R}^{3}(3 \mathrm{a}-\mathrm{d})$. This reaction is formally equivalent to an exchange of the hydrogen bound to silicon and the acyloxy group bound to carbon. The 1,2-hydrogen shift could be shown experimentally to be an intramolecular process.

Mit dem Ziel, weiteren Aufschluß über das chemische Verhalten der kürzlich von uns beschriebenen ${ }^{1)}$ (Hydroxymethyl)diorganylsilane 1a - c zu erhalten, haben wir diese difunktionellen Verbindungen mit Essigsäureanhydrid (1 b, c), mit Acetylchlorid und Pyridin (1a, b) bzw. mit Benzoylchlorid und Pyridin (1a) umgesetzt. Unter Einhaltung bestimmter Bedingungen entstehen hierbei durch Reaktion an den alkoholischen $\mathrm{OH}-$ Gruppen in guten Ausbeuten die entsprechenden (Acyloxymethyl)diorganylsilane 2a-d.

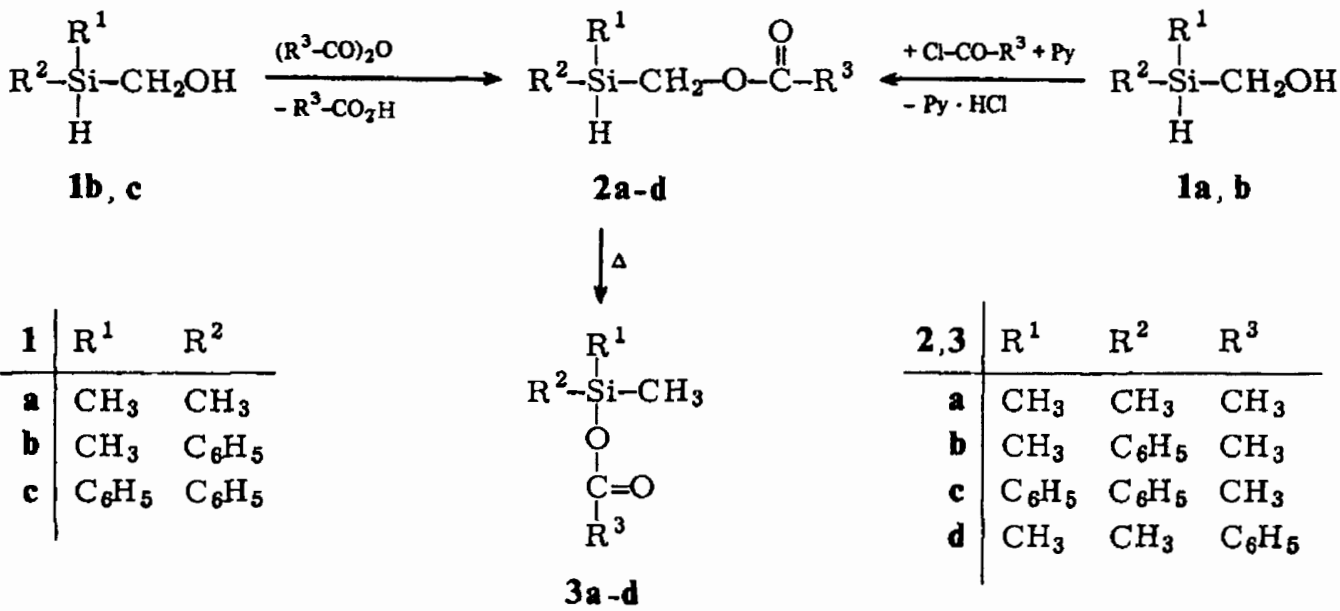

(C) Verlag Chemie GmbH, D-6940 Weinheim, 1983 
Diese erwiesen sich zu unserer Überraschung als thermisch nicht sehr stabil: Sie gehen beim Erhitzen - sowohl in Substanz als auch in benzolischer Lösung - in die entsprechenden Acyloxy(methyl)diorganylsilane 3a-d über; eine Umlagerung, die formal einen Austausch des am Silicium gebundenen Wasserstoffs mit dem am Kohlenstoff gebundenen Acyloxy-Rest beinhaltet*).

Kinetische Untersuchungen ergaben, daß diese Umwandlungen dem Geschwindigkeitsgesetz 1. Ordnung folgen. Bei $135^{\circ} \mathrm{C}$ wurden Halbwertszeiten im Bereich von ca. 3-12 $\mathrm{h}$ gemessen, wobei sich bezüglich der Umlagerungsgeschwindigkeiten - gemessen an benzolischen Lösungen der Substanzen - folgende Reihenfolge ergab: 2c > 2d $>2$ b $>2$ a (Tab. 1). Am Beispiel der Reaktion von $2 \mathrm{c}$ zu 3c wurde eine Aktivierungsenergie von $E_{\mathrm{A}} \approx 27 \mathrm{kcal} / \mathrm{mol}$ bestimmt.

Tab. 1. Geschwindigkeitskonstanten $k$ der Umlagerung von $2 \mathbf{a}-\mathbf{e}$ in $\mathbf{3 a}-\mathbf{e}$

\begin{tabular}{cccc}
\hline Umlagerung & $\begin{array}{c}\text { Losungs- } \\
\text { mittel }\end{array}$ & $\begin{array}{c}\text { Temp. } \\
{\left[{ }^{\circ} \mathrm{C}\right]}\end{array}$ & $\begin{array}{c}k \times 10^{5} \\
{\left[\mathbf{s}^{-1}\right]}\end{array}$ \\
\hline $\mathbf{2 a} \rightarrow \mathbf{3 a}$ & $\mathrm{C}_{6} \mathrm{D}_{6}$ & 135 & 1.7 \\
$\mathbf{2 b} \rightarrow \mathbf{3 b}$ & $\mathrm{C}_{6} \mathrm{D}_{6}$ & 135 & 2.5 \\
$\mathbf{2 b} \rightarrow \mathbf{3 b}$ & ohne & 135 & 4.0 \\
$\mathbf{2} \mathbf{c} \rightarrow \mathbf{3 c}$ & $\mathrm{C}_{6} \mathrm{D}_{6}$ & 150 & 15 \\
$\mathbf{2 c} \rightarrow \mathbf{3 c}$ & $\mathrm{C}_{6} \mathrm{D}_{6}$ & 135 & 4.6 \\
$\mathbf{2 c} \rightarrow \mathbf{3 c}$ & $\mathrm{C}_{6} \mathrm{D}_{6}$ & 110 & 0.52 \\
$\mathbf{2 c} \rightarrow \mathbf{3 c}$ & ohne & 135 & 6.7 \\
$\mathbf{2 d} \rightarrow \mathbf{3 d}$ & $\mathrm{C}_{6} \mathrm{D}_{6}$ & 135 & 3.0 \\
$\mathbf{2 e} \rightarrow \mathbf{3 e}$ & $\mathrm{C}_{6} \mathrm{D}_{6}$ & 135 & $\mathbf{2 . 6}$ \\
\hline
\end{tabular}

Bezüglich des einen der beiden formalen Teilschritte - nämlich der 1,2-WasserstoffVerschiebung ${ }^{2)}$ - ließ sich durch Kreuzungsexperimente ein intramolekularer Prozeß beweisen: Gemeinsames Erhitzen des Silans 2b und der Deuterium-markierten Verbindung $2 \mathrm{e}$ - zugänglich durch Umsetzung von 1d mit Essigsäureanhydrid - führte ausschließlich zu den Produkten 3b und 3e; das Deuterium ließ sich nur in der Methylgruppe des Silans 3e nachweisen, welches uns als authentische Vergleichssubstanz dargestellt durch Erhitzen von $2 \mathrm{e}$ - zur Verfügung stand. Aus den bei $135^{\circ} \mathrm{C}$ bestimmten Geschwindigkeitskonstanten der Umlagerung der beiden Isotopomere $2 \mathrm{c}$ und $2 \mathrm{e}$ (vgl. Tab. 1) errechnet sich ein kinetischer Isotopeneffekt von $k_{\mathrm{H}} / k_{\mathrm{D}} \approx 1.8$; dieser erscheint jedoch zu klein, um ihn zur Deutung der mechanistischen Verhältnisse mit heranzuziehen.

*) Anmerkung bei der Korr. (17.5. 1983): Kürzlich wurde eine radikalisch initiierte Umlagerung von $2 \mathrm{a}$ in $\mathbf{3 a}$ in der Literatur $[J . W$. Wilt und S. M. Keller, J. Am. Chem. Soc. 105, 1395 (1983)] beschrieben; in der gleichen Arbeit wird darauf hingewiesen, daß 2a auch einer thermisch induzierten Umlagerung in 3a unterliegt - wie auch von uns gefunden, bereits an anderer Stelle vorpubliziert $[R$. Tacke, $H$. Lange und $A$. Bentlage, Chemiedozententagung, Kaiserslautern 1982, Vortragsreferate, S. 118; Lit. ${ }^{1}$ ] und in der vorliegenden Arbeit ausführlich beschrieben. - Uber die analoge Umlagerung von $2 \mathbf{b}$ in 3b wurde bereits zuvor berichtet $\left[R\right.$. Tacke, M. T. Attar-Bashi, A. Bentlage und H. Lange, $6^{\text {th }}$ International Symposium on Organosilicon Chemistry, Budapest 1981, Abstracts of Papers, S. 204].

Chem. Ber. $116(1983)$ 
Ein ebenfalls denkbarer intramolekularer Transfer des Acyloxy-Restes als zweiter formaler Teilschritt der Umlagerung konnte durch entsprechende Kreuzungsexperimente mit $2 \mathrm{c}$ und der Deuterium-markierten Verbindung $2 \mathbf{f}$ - erhältlich durch Umsetzung von $1 \mathrm{~b}$ mit $\left[\mathrm{D}_{6}\right]$ Essigsäureanhydrid - nicht bewiesen werden: Gemeinsames Erhitzen äquivalenter Mengen von 2c und $2 \mathrm{f}$ führte zu einem 1:1:1:1-Gemisch (NMRspektroskopisch) von 3b, 3c, 3f und 3g. Dieses Ergebnis darf jedoch nicht als Beweis für eine intermolekulare Wanderung des Acyloxy-Restes gewertet werden, da Kontrolluntersuchungen zeigten, daß $3 \mathbf{c}$ und $\mathbf{3 f}$ bereits unter milden Bedingungen einem intermolekularen Acetoxy-Austausch zu $3 \mathrm{~g}$ und $\mathbf{3 b}$ (und umgekehrt) unterliegen ${ }^{3)}$ : In Lösung $\left[\mathrm{CCl}_{4} /\left[\mathrm{D}_{12}\right]\right.$ Cyclohexan(9:1)] erfolgt die Gleichgewichtseinstellung bei Raumtemperatur im Verlauf einiger Tage, ohne Lösungsmittel bereits innerhalb weniger Minuten.

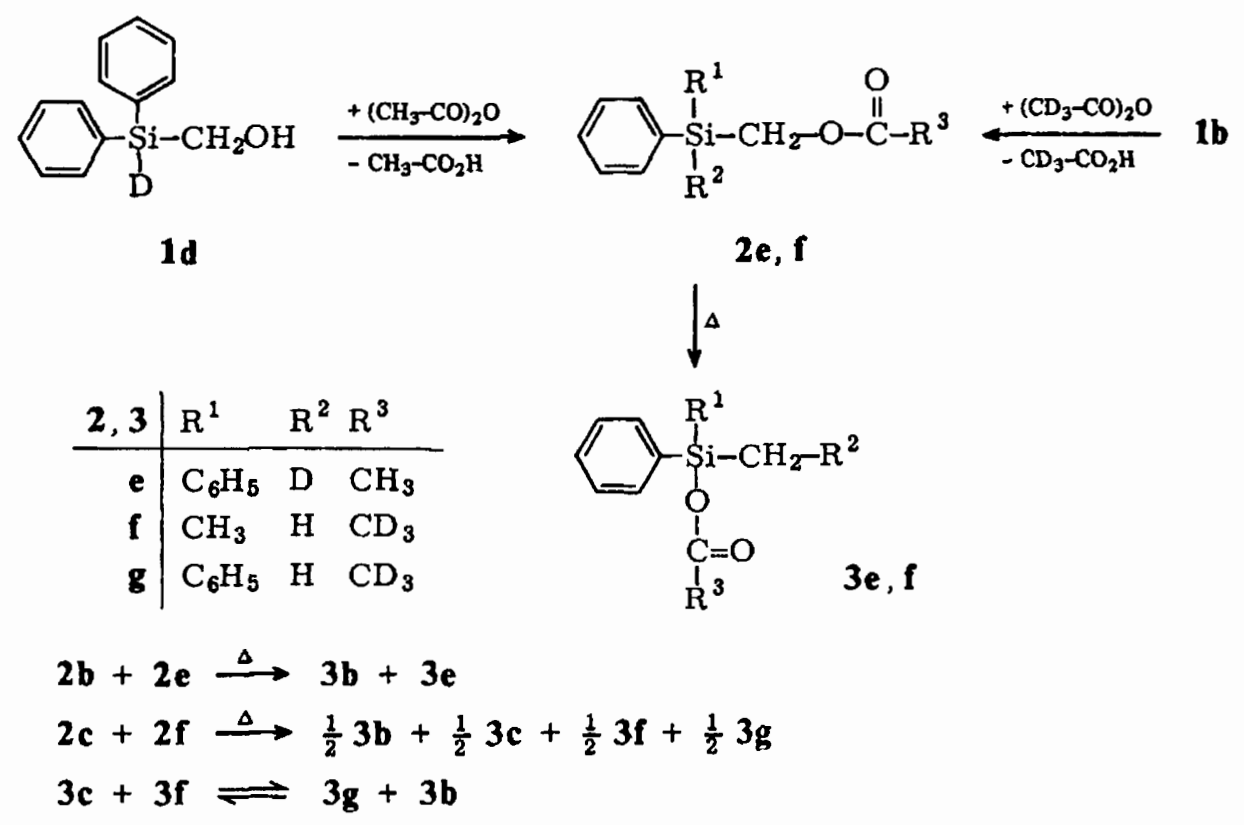

Die hier vorgestellte Umlagerung von (Acyloxymethyl)diorganylsilanen erinnert zumindest bei formaler Betrachtungsweise - an Umlagerungsreaktionen des allgemeinen Typs $R_{2}(X) S_{C i C R} Y \rightarrow R_{2}(Y) S_{2} R_{2} X$, die unlängst bei einigen anderen Silanen aufgefunden wurden ${ }^{4-10)}$. Inwieweit es sich hierbei tatsächlich um ein mechanistisch einheitliches Reaktionsprinzip handelt, ist derzeit eine noch offene Frage, deren Beantwortung weiterer Untersuchungen bedarf. In diesem Zusammenhang sei auch auf die $\mathrm{AlCl}_{3}$-katalysierte Umwandlung von Verbindungen des Typs $\mathrm{R}_{2}(\mathrm{H}) \mathrm{SiCHCl}_{2}$ in $\mathbf{R}_{\mathbf{2}}(\mathrm{Cl})$ $\mathrm{SiCH}_{2} \mathrm{Cl}$ hingewiesen ${ }^{16)}$.

Prăparative Studien unsererseits haben inzwischen gezeigt, daß die an den Silanen $2 \mathfrak{a}-\mathbf{f}$ beobachteten Umlagerungsreaktionen nicht auf (Diorganylsilyl)methyl-ester von Carbonsäuren beschränkt sind. Wir fanden, daß auch entsprechende Ester bestimmter anorganischer Elementsauerstoffsäuren analog reagieren: So lagert sich 2. B. der (Dimethylsilyl)methyl-substituierte Phosphorsäureester 4 beim Erwärmen zu dem Trimethylsilyl-Derivat 5 um $^{11)}$, und die (Methylphenylsilyl)methyl-substituierten Kohlensäureester-Derivate 6a-c ergeben beim Erhitzen die Silane $7 \mathbf{a}-\mathbf{c}^{12}$, wobei die Bildung der drei letztgenannten Verbindungen neben der Umlagerung noch eine zusätzliche Decarboxylierung beinhaltet.

Chem. Ber. $116(1983)$ 


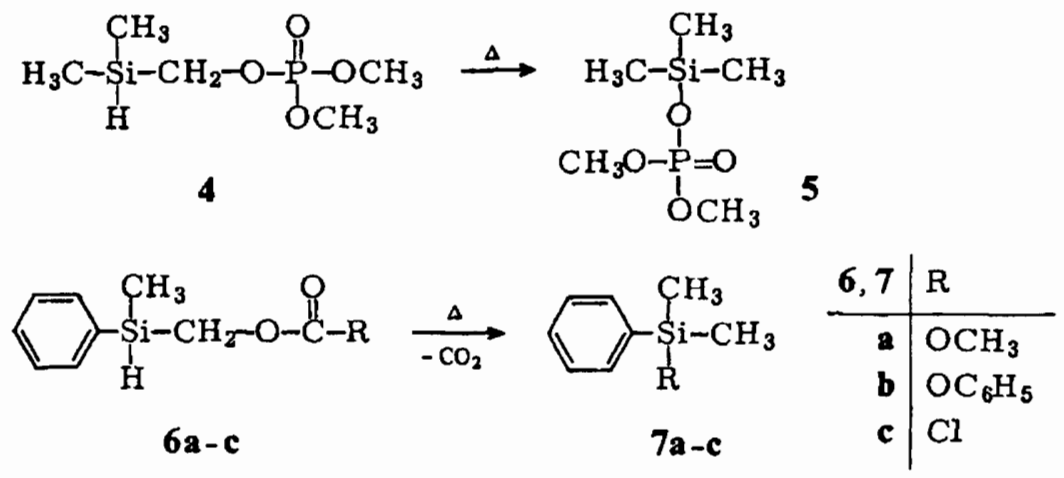

Gegenwärtige Untersuchungen dienen dem Ziel, das präparative Potential des hier vorgestellten Umlagerungsprinzips auszuloten. Dabei ist nicht nur an Anwendungen im Bereich der Anorganischen Chemie - speziell der Siliciumchemie - zu denken; ebenso könnten sich auch interessante Möglichkeiten für die Organische Synthese bieten. So untersuchen wir z. B. die potentielle Verwendbarkeit von $\mathbf{R}_{2}(\mathrm{H}) \mathrm{SiCH}_{2}$-Resten ( $\mathrm{R}=$ Organyl) als Schutzgruppen für Carboxyl-Funktionen: $\mathbf{R}_{\mathbf{2}}(\mathrm{H}) \mathrm{SiCH}_{2}$-geschützte Carbonsäuren 8 sollten in bestimmten Medien - so z. B. auch in sauren wasserhaltigen Systemen - einer Reihe chemischer Reaktionen am Rest $R^{1}$ zugänglich sein $(8 \rightarrow 9)$, ohne daß hierbei die $\mathrm{Si}-\mathrm{H}$-Funktion der Schutzgruppe zerstört wird. Anschließend wäre durch thermische Behandlung von 9 grundsätzlich eine Uberführung in die $\mathrm{R}_{2}\left(\mathrm{CH}_{3}\right) \mathrm{Si}$ substituierten Derivate 12 möglich, die in der Regel problemlos zu den entsprechenden Silanolen 10 und Carbonsăuren 11 hydrolysiert werden können.

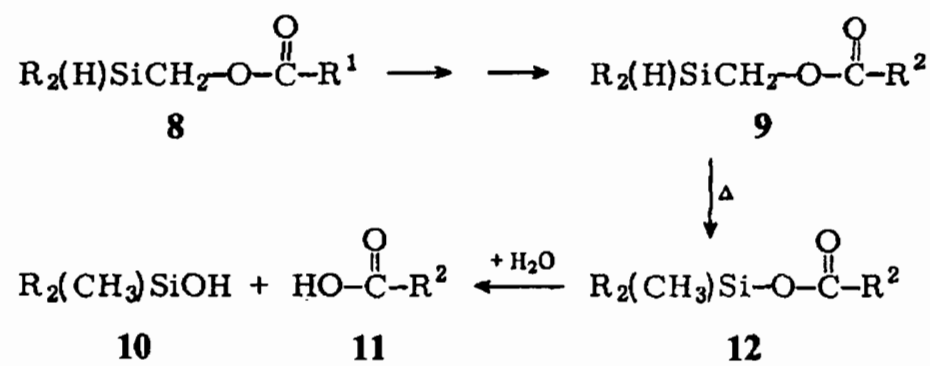

Unser Dank gilt der Deutschen Forschungsgemeinschaft und dem Fonds der Chemischen Industrie für die Unterstützung dieser Arbeit. Der Bayer AG, Wuppertal-Elberfeld und Leverkusen, danken wir für die Bereitstellung von Chemikalien, Herrn Priv.-Doz. Dr. L. Ernst für einige NMR-spektroskopische Messungen.

\section{Experimenteller Teil}

Brechungsindices: Abbé-Refraktometer (Fa. Zeiss). - ${ }^{1} \mathrm{H}-\mathrm{NMR}$-Spektren ( $90 \mathrm{MHz}$, int. Standard TMS): HFX-90-Gerăt (Fa. Bruker). $-{ }^{13} \mathrm{C}$-NMR-Spektren [100.6 MHz, int. Standard $\left.\mathrm{CDCl}_{3}(\delta=77.05)\right]$ : WM-400-Gerät (Fa. Bruker). - IR-Spektren: AccuLab-9-Gerăt (Fa. Beckman). - Massenspektren (70 eV, angegeben sind ausgesuchte, strukturbeweisende Fragmente): CH-7-Gerät (Fa: Atlas MAT). - Bei den kinetischen Messungen wurde mit einem BadThermostaten D3-19 (Fa. Haake) eine Temperaturkonstanz von $\pm 0.02^{\circ} \mathrm{C}$ sichergestellt. Die Kinetik wurde ${ }^{1} \mathrm{H}$-NMR-spektroskopisch verfolgt (R-24B-Gerät, $60 \mathrm{MHz}, \mathrm{Fa}$. Hitachi-PerkinElmer). - Alle Reaktionen wurden unter einer Schutzgasatmosphäre von trockenem Stickstoff und - sofern nicht anders angegeben - in wasserfreien Lơsungsmitteln durchgeführt.

Ausgangsverbindungen: (Hydroxymethyl)dimethylsilan (1 a), (Hydroxymethyl)methylphenylsilan (1 b) und (Hydroxymethyl)diphenylsilan (1c) wurden gemäß Lit. ${ }^{1)}$ hergestellt. - Deuterio(hydroxymethyl)diphenylsilan (1d): Analog zu 1a durch Umsetzung von $22.01 \mathrm{~g}$ (70 mmol) 
Acetoxy(acetoxymethyl)diphenylsilan ${ }^{1)}$ mit $4.20 \mathrm{~g}(0.1 \mathrm{~mol}) \mathrm{LiAlD}_{4}$ (Merck, Art. 5664, Deuterierungsgrad >98\%). Ausb. $9.8 \mathrm{~g}(65 \%)$ einer farblosen Flüssigkeit vom Sdp. $125^{\circ} \mathrm{C} / 0.1$ Torr. ${ }^{1} \mathrm{H}$-NMR $\left(\mathrm{CCl}_{4}\right): \delta=3.65\left(\mathrm{~s},{ }^{3} \mathrm{~J}_{\mathrm{HD}}\right.$ nicht aufgelöst, $\left.2 \mathrm{H} ; \mathrm{SiCH}_{2} \mathrm{O}\right), 2.3$ (s, verbreitert, nicht lagekonstant, $1 \mathrm{H} ; \mathrm{OH}), 7.1-7.7\left(\mathrm{~m}, 10 \mathrm{H} ; \mathrm{SiC}_{6} \mathrm{H}_{5}\right)$. - IR (Film): $3560(\mathrm{O}-\mathrm{H}$, nichtassoziiert), 3350 (Max.; $\mathrm{O}-\mathrm{H}$, assoziiert), $1550 \mathrm{~cm}^{-1}(\mathrm{Si}-\mathrm{D})$.

(Acetoxymethyl)dimethylsilan (2a): Zu einer Lösung von $9.02 \mathrm{~g}(100 \mathrm{mmol}) 1 \mathrm{a}$ und $7.91 \mathrm{~g}$ (100 mmol) Pyridin in $800 \mathrm{ml}$ Pentan wurden bei $-70^{\circ} \mathrm{C}$ innerhalb von 30 min unter Ruhren $7.85 \mathrm{~g}$ $(100 \mathrm{mmol})$ Acetylchlorid in $100 \mathrm{ml}$ Pentan getropft. Man ließ $20 \mathrm{~h}$ rühren und währenddessen auf Raumtemp. erwärmen, filtrierte, befreite das Filtrat unter vermindertem Druck vom Lösungsmittel und unterwarf den Rückstand einer säulenchromatographischen Grobreinigung an $\mathrm{Al}_{2} \mathrm{O}_{3}$ ( $\approx 100 \mathrm{~g} \mathrm{Al}_{2} \mathrm{O}_{3} 90$ aktiv, neutral, Merck Art. 1077; Eluens Pentan). Die fraktionierende Vakuumdestillation des Eluats uber eine Vigreux-Kolonne lieferte $9.3 \mathrm{~g}(70 \%)$ einer farblosen Flüssigkeit, Sdp. $55-57^{\circ} \mathrm{C} / 50$ Torr, $n_{\mathrm{D}}^{20}=1.411$. - IR (Film): $2120(\mathrm{Si}-\mathrm{H}), 1740 \mathrm{~cm}^{-1}$ $(\mathrm{C}=\mathrm{O}) .-{ }^{1} \mathrm{H}-\mathrm{NMR}\left(\mathrm{CCl}_{4}\right): \delta=0.14\left(\mathrm{~d},{ }^{3} \mathrm{~J}_{\mathrm{HH}}=3.5 \mathrm{~Hz}, 6 \mathrm{H} ; \mathrm{SiCH}_{3}\right), 2.00\left(\mathrm{~s}, 3 \mathrm{H} ; \mathrm{CCH}_{3}\right), 3.81$ $\left(\mathrm{d},{ }^{3} J_{\mathrm{HH}}=2 \mathrm{~Hz}, 2 \mathrm{H} ; \mathrm{SiCH}_{2} \mathrm{O}\right), 3.99(\mathrm{tsept}, 1 \mathrm{H} ; \mathrm{SiH}) .-\mathrm{MS}: m / e=131\left(57 \%, \mathrm{M}^{+}-\mathrm{H}\right), 117$ $\left(60 \%, \mathrm{M}^{+}-\mathrm{CH}_{3}\right), 89\left(76 \%, \mathrm{M}^{+}-\mathrm{C}(\mathrm{O}) \mathrm{CH}_{3}\right), 59\left(83 \%, \mathrm{M}^{+}-\mathrm{CH}_{2} \mathrm{OC}(\mathrm{O}) \mathrm{CH}_{3}\right), 43(100 \%$, $\mathrm{CH}_{3} \mathrm{CO}^{+}$und $\mathrm{CH}_{3} \mathrm{Si}^{+}$).

$$
\mathrm{C}_{5} \mathrm{H}_{12} \mathrm{O}_{2} \mathrm{Si} \text { (132.2) Ber. C } 45.42 \text { H 9.15 Si } 21.24 \text { Gef. C } 45.6 \text { H } 9.15 \text { Si } 21.0
$$

(Acetoxymethyl)methylphenylsilan (2b); Methode a: Zu einer Lösung von $3.05 \mathrm{~g}(20 \mathrm{mmol}) \mathbf{1 b}$ und $3.1 \mathrm{~g}(39 \mathrm{mmol})$ Pyridin in $50 \mathrm{ml}$ Petrolether $\left(35-70^{\circ} \mathrm{C}\right)$ wurde bei $-30^{\circ} \mathrm{C}$ unter Rühren innerhalb von $30 \mathrm{~min}$ eine Lösung von $1.57 \mathrm{~g}(20 \mathrm{mmol})$ Acetylchlorid in $20 \mathrm{ml}$ Petrolether getropft. Nach 2stündigem Rühren bei dieser Temp. ließ man auf $20^{\circ} \mathrm{C}$ erwärmen, verdampfte das Lösungsmittel und nicht umgesetztes Pyridin i.Vak. und destillierte den Rückstand im Kugelrohrverdampfer. Ausb. $2.8 \mathrm{~g}(\mathbf{7 2 \%})$ eines ${ }^{1} \mathrm{H}$-NMR-spektroskopisch reinen Produktes.

Methode $b$ : Eine Mischung aus $7.61 \mathrm{~g}(50 \mathrm{mmol}) 1 \mathrm{~b}$ und $6.13 \mathrm{~g}(60 \mathrm{mmol})$ Essigsäureanhydrid wurde $1 \mathrm{~h}$ bei $60^{\circ} \mathrm{C}$ gerührt. Die leichtflüchtigen Anteile wurden unter vermindertem Druck verdampft und der Rückstand i. Vak. über eine Vigreux-Kolonne fraktionierend destilliert. Ausb. $8.3 \mathrm{~g}(85 \%)$ einer farblosen Flüssigkeit, Sdp. $87^{\circ} \mathrm{C} / 0.05$ Torr, $n_{\mathrm{D}}^{20}=1.506$. - IR (Film): 2130 $(\mathrm{Si}-\mathrm{H}), 1740 \mathrm{~cm}^{-1}(\mathrm{C}=\mathrm{O}) .-{ }^{1} \mathrm{H}-\mathrm{NMR}\left(\mathrm{CCl}_{4}\right): \delta=0.40\left(\mathrm{~d},{ }^{3} \mathrm{~J}_{\mathrm{HH}}=4 \mathrm{~Hz}, 3 \mathrm{H} ; \mathrm{SiCH}_{3}\right), 1.93(\mathrm{~s}$, $\left.3 \mathrm{H} ; \mathrm{CCH}_{3}\right), 3.96\left(\mathrm{~d},{ }^{3} J_{\mathrm{HH}}=2.5 \mathrm{~Hz}, 2 \mathrm{H} ; \mathrm{SiCH}_{2} \mathrm{O}\right), 4.47(\mathrm{tq}, 1 \mathrm{H} ; \mathrm{SiH}), 7.2-7.6(\mathrm{~m}, 5 \mathrm{H}$; $\left.\mathrm{SiC}_{6} \mathrm{H}_{5}\right) .-\mathrm{MS}: m / e=193\left(62 \%, \mathrm{M}^{+}-\mathrm{H}\right), 179\left(72 \%, \mathrm{M}^{+}-\mathrm{CH}_{3}\right), 151\left(68 \%, \mathrm{M}^{+}-\right.$ $\left.\mathrm{C}(\mathrm{O}) \mathrm{CH}_{3}\right), 121\left(100 \%, \mathrm{M}^{+}-\mathrm{CH}_{2} \mathrm{OC}(\mathrm{O}) \mathrm{CH}_{3}\right), 117\left(95 \%, \mathrm{M}^{+}-\mathrm{C}_{6} \mathrm{H}_{5}\right), 43\left(90 \%, \mathrm{CH}_{3} \mathrm{CO}^{+}\right.$ und $\mathrm{CH}_{3} \mathrm{Si}^{+}$).

$$
\mathrm{C}_{10} \mathrm{H}_{14} \mathrm{O}_{2} \mathrm{Si} \text { (194.3) Ber. C } 61.82 \text { H 7.26 Si 14.45 Gef. C } 61.8 \text { H 7.4 Si 14.3 }
$$

(Acetoxymethyl)diphenylsilan (2c): Analog zur Darstellung von $2 \mathrm{~b}$ gemäß Methode $b$ durch Umsetzung von $10.72 \mathrm{~g} \mathrm{(50} \mathrm{mmol)} 1 \mathrm{c}$ mit $6.13 \mathrm{~g} \mathrm{(60} \mathrm{mmol)} \mathrm{Essigsäureanhydrid.} \mathrm{Reinigung} \mathrm{durch}$ zweimalige Destillation im Kugelrohrverdampfer ergab $11.3 \mathrm{~g}(\mathbf{8 8 \%})$ einer farblosen Flüssigkeit, die als Verunreinigung etwa $3 \%$ ( $\left.{ }^{1} \mathrm{H}-\mathrm{NMR}\right) 3 \mathrm{c}$ enthielt. Sdp. $112-114^{\circ} \mathrm{C} / 0.1 \mathrm{Torr}, n_{\mathrm{D}}^{20}=1.556$. - IR (Film): 2135 ( $\mathrm{Si}-\mathrm{H}), 1730 \mathrm{~cm}^{-1}(\mathrm{C}=\mathrm{O}) .-{ }^{1} \mathrm{H}-\mathrm{NMR}\left(\mathrm{CCl}_{4}\right): \delta=1.84\left(\mathrm{~s}, 3 \mathrm{H} ; \mathrm{CCH}_{3}\right), 4.21$ $\left(\mathrm{d},{ }^{3} J_{\mathrm{HH}}=3 \mathrm{~Hz}, 2 \mathrm{H} ; \mathrm{SiCH}_{2} \mathrm{O}\right), 4.96\left(\mathrm{t},{ }^{3} J_{\mathrm{HH}}=3 \mathrm{~Hz}, 1 \mathrm{H} ; \mathrm{SiH}\right), 7.1-7.7\left(\mathrm{~m}, 10 \mathrm{H} ; \mathrm{SiC}_{6} \mathrm{H}_{5}\right)$. MS: $m / e=255\left(41 \%, \mathrm{M}^{+}-\mathrm{H}\right), 213\left(43 \%, \mathrm{M}^{+}-\mathrm{C}(\mathrm{O}) \mathrm{CH}_{3}\right), 183\left(79 \%, \mathrm{M}^{+}-\right.$ $\left.\mathrm{CH}_{2} \mathrm{OC}(\mathrm{O}) \mathrm{CH}_{3}\right), 179\left(100 \%, \mathrm{M}^{+}-\mathrm{C}_{6} \mathrm{H}_{5}\right), 43\left(63 \%, \mathrm{CH}_{3} \mathrm{CO}^{+}\right)$.

$$
\mathrm{C}_{15} \mathrm{H}_{16} \mathrm{O}_{2} \mathrm{Si} \text { (256.4) Ber. C } 70.27 \text { H 6.29 Si } 10.95 \text { Gef. C } 70.5 \text { H } 6.4 \text { Si } 11.1
$$

(Benzoyloxymethyl)dimethylsilan (2d): Analog zur Darstellung von 2a durch Umsetzung einer Lösung von $9.02 \mathrm{~g}(100 \mathrm{mmol}) 1 \mathrm{a}$ und $7.91 \mathrm{~g}(100 \mathrm{mmol})$ Pyridin in $800 \mathrm{ml}$ Pentan mit einer Lösung von $14.06 \mathrm{~g}$ (100 mmol) Benzoylchlorid in $100 \mathrm{ml}$ Pentan. Ausb. $13.0 \mathrm{~g}(67 \%)$ einer farblo- 
sen Flüssigkeit, Sdp. $50-52^{\circ} \mathrm{C} / 0.01$ Torr, $n_{\mathrm{D}}^{20}=1.503$. - IR (Film): $2120(\mathrm{Si}-\mathrm{H}), 1720 \mathrm{~cm}^{-1}$ $(\mathrm{C}=0) .-{ }^{1} \mathrm{H}-\mathrm{NMR}\left(\mathrm{CCl}_{4}\right): \delta=0.22\left(\mathrm{~d},{ }^{3} \mathrm{~J}_{\mathrm{HH}}=3.5 \mathrm{~Hz}, 6 \mathrm{H} ; \mathrm{SiCH}_{3}\right), 4.07\left(\mathrm{~d},{ }^{3} \mathrm{~J}_{\mathrm{HH}}=1.5 \mathrm{~Hz}\right.$, $2 \mathrm{H}$; $\mathrm{SiCH}_{2} \mathrm{O}$; uberlagert durch tsept bei 4.1), 4.1 (tsept, $1 \mathrm{H}$; $\mathrm{SiH}$; ubberlagert durch d bei 4.07), 7.3-7.6 und 7.9-8.1 (m, $\left.5 \mathrm{H} ; \mathrm{CC}_{6} \mathrm{H}_{5}\right)$. $-\mathrm{MS}: m / e=193\left(78 \%, \mathrm{M}^{+}-\mathrm{H}\right), 179\left(57 \%, \mathrm{M}^{+}-\right.$ $\left.\mathrm{CH}_{3}\right), \quad 105\left(100 \%, \mathrm{C}_{6} \mathrm{H}_{5} \mathrm{CO}^{+}\right), 89\left(44 \%, \mathrm{M}^{+}-\mathrm{C}(\mathrm{O}) \mathrm{C}_{6} \mathrm{H}_{5}\right), 59\left(58 \%, \mathrm{M}^{+}-\right.$ $\left.\mathrm{CH}_{2} \mathrm{OC}(\mathrm{O}) \mathrm{C}_{6} \mathrm{H}_{5}\right)$.

$$
\mathrm{C}_{10} \mathrm{H}_{14} \mathrm{O}_{2} \mathrm{Si}(194.3) \text { Ber. C } 61.82 \text { H 7.26 Si 14.45 Gef. C } 61.7 \text { H 7.3 Si 14.4 }
$$

(Acetoxymethyl)deuteriodiphenylsilan (2e): Analog zur Darstellung von 2c durch Umsetzung von $8.61 \mathrm{~g}(40 \mathrm{mmol}) 1 \mathrm{~d}$ mit $5.10 \mathrm{~g}(50 \mathrm{mmol})$ Essigsăureanhydrid. Ausb. $8.95 \mathrm{~g} \mathrm{(87 \% )}$. - IR (Film): $1735(\mathrm{C}=0), 1560 \mathrm{~cm}^{-1}(\mathrm{Si}-\mathrm{D}) .-{ }^{1} \mathrm{H}-\mathrm{NMR}\left(\mathrm{CCl}_{4}\right): \delta=1.88\left(\mathrm{~s}, 3 \mathrm{H} ; \mathrm{CCH}_{3}\right), 4.24(\mathrm{~s}$, ${ }^{3} J_{\mathrm{HD}}$ nicht aufgelost, $\left.2 \mathrm{H} ; \mathrm{SiCH}_{2} \mathrm{O}\right), 7.1-7.7\left(\mathrm{~m}, 10 \mathrm{H} ; \mathrm{SiC}_{6} \mathrm{H}_{\mathrm{g}}\right) .-\mathrm{MS}: m / e=255(33 \%$, $\left.\mathrm{M}^{+}-\mathrm{D}\right), 214\left(8 \%, \mathrm{M}^{+}-\mathrm{C}(\mathrm{O}) \mathrm{CH}_{3}\right), 184\left(74 \%, \mathrm{M}^{+}-\mathrm{CH}_{2} \mathrm{OC}(\mathrm{O}) \mathrm{CH}_{3}\right), 180\left(100 \%, \mathrm{M}^{+}-\right.$ $\left.\mathrm{C}_{6} \mathrm{H}_{5}\right), 43\left(66 \%, \mathrm{CH}_{3} \mathrm{CO}^{+}\right)$. $-\mathrm{C}_{15} \mathrm{H}_{15} \mathrm{DO}_{2} \mathrm{Si}(257.4)$.

$\left\{\left(\left[D_{3}\right]\right.\right.$ Acetoxy)methyl $\}$ methylphenylsilan (2f): Analog zur Darstellung von 2b gemäß Methode $b$ durch Umsetzung von $6.09 \mathrm{~g}(40 \mathrm{mmol}) 1 \mathrm{~b}$ mit $5.41 \mathrm{~g}(50 \mathrm{mmol})\left[\mathrm{D}_{6}\right]$ Essigsäureanhydrid (EGA-Chemie, Art. 17,546-1, Deuterierungsgrad $>99 \%$ ). Ausb. $6.45 \mathrm{~g}(82 \%)$. - ${ }^{1} \mathrm{H}$-NMR $\left(\mathrm{CCl}_{4}\right): \delta=0.42\left(\mathrm{~d},{ }^{3} J_{\mathrm{HH}}=4 \mathrm{~Hz}, 3 \mathrm{H} ; \mathrm{SiCH}_{3}\right), 3.98\left(\mathrm{~d},{ }^{3} J_{\mathrm{HH}}=2.5 \mathrm{~Hz}, 2 \mathrm{H} ; \mathrm{SiCH}_{2} \mathrm{O}\right), 4.48(\mathrm{tq}$, $1 \mathrm{H} ; \mathrm{SiH}), 7.2-7.6\left(\mathrm{~m}, 5 \mathrm{H} ; \mathrm{SiC}_{6} \mathrm{H}_{5}\right) .-\mathrm{MS}: m / e=196\left(43 \%, \mathrm{M}^{+}-\mathrm{H}\right), 182\left(46 \%, \mathrm{M}^{+}-\right.$ $\left.\mathrm{CH}_{3}\right), 151\left(36 \%, \mathrm{M}^{+}-\mathrm{C}(\mathrm{O}) \mathrm{CD}_{3}\right), 121\left(100 \%, \mathrm{M}^{+}-\mathrm{CH}_{2} \mathrm{OC}(\mathrm{O}) \mathrm{CD}_{3}\right), 119\left(52 \%, \mathrm{M}^{+}-\right.$ $\left.\mathrm{C}_{6} \mathrm{H}_{5}\right), 46\left(98 \%, \mathrm{CD}_{3} \mathrm{CO}^{+}\right)$. $-\mathrm{C}_{10} \mathrm{H}_{11} \mathrm{D}_{3} \mathrm{O}_{2} \mathrm{Si}$ (197.3).

Umlagerung von $2 \mathrm{a}-\mathrm{f} z u \mathbf{3 a}-\mathrm{f}: 2.00 \mathrm{~g}(15.1 \mathrm{mmol}) 2 \mathrm{a}$ wurden $48 \mathrm{~h}$ auf Siedehitze (Normaldruck) erwärmt; $2.00 \mathrm{~g}(10.3 \mathrm{mmol}) 2 \mathrm{~b}, 2.00 \mathrm{~g}(7.8 \mathrm{mmol}) 2 \mathrm{c}, 2.00 \mathrm{~g}(10.3 \mathrm{mmol}) 2 \mathrm{~d}, 2.00 \mathrm{~g}(7.8$ mmol) $2 \mathrm{e}$ bzw. $2.00 \mathrm{~g}(10.1 \mathrm{mmol}) 2 \mathrm{f}$ wurden $4 \mathrm{~h}$ unter vermindertem Druck auf $180^{\circ} \mathrm{C}$ gehalten. Die ${ }^{1} \mathrm{H}$-NMR-spektroskopisch einheitlichen Produkte wurden für analytische Zwecke über eine Vigreux-Kolonne i. Vak. destilliert.

Acetoxytrimethylsilan (3a): Ausb. $1.8 \mathrm{~g}(90 \%)$, Sdp. $48-50^{\circ} \mathrm{C} / 50$ Torr (Lit. ${ }^{13)}$ Sdp. $44-45^{\circ} \mathrm{C} / 79$ Torr), $n_{\mathrm{D}}^{20}=1.388$ (Lit. $\left.{ }^{13)} n_{\mathrm{D}}^{20}=1.3890\right)$. - IR (Film): $1720 \mathrm{~cm}^{-1}(\mathrm{C}=0)$. ${ }^{1} \mathrm{H}-\mathrm{NMR}\left(\mathrm{CCl}_{4}\right): \delta=0.26\left(\mathrm{~s}, 9 \mathrm{H} ; \mathrm{SiCH}_{3}\right), 2.01\left(\mathrm{~s}, 3 \mathrm{H} ; \mathrm{CCH}_{3}\right) .-\mathrm{MS}: m / e=132\left(6 \%, \mathrm{M}^{+}\right)$.

Acetoxydimethylphenylsilan (3b): Ausb. $1.6 \mathrm{~g}(80 \%)$, Sdp. $67-69^{\circ} \mathrm{C} / 0.05$ Torr (Lit. ${ }^{14)} \mathrm{Sdp}$. $92-95^{\circ} \mathrm{C} / 7$ Torr), $n_{\mathrm{D}}^{20}=1.491$ (Lit. $\left.{ }^{14)} n_{\mathrm{D}}^{20}=1.4907\right)$. - IR (Film): $1720 \mathrm{~cm}^{-1}(\mathrm{C}=0)$. ${ }^{1} \mathrm{H}-\mathrm{NMR}\left(\mathrm{CCl}_{4}\right): \delta=0.53\left(\mathrm{~s}, 6 \mathrm{H} ; \mathrm{SiCH}_{3}\right), 2.00\left(\mathrm{~s}, 3 \mathrm{H} ; \mathrm{CCH}_{3}\right), 7.2-7.7\left(\mathrm{~m}, 5 \mathrm{H} ; \mathrm{SiC}_{6} \mathrm{H}_{5}\right)$. MS: $m / e=194\left(3 \%, \mathrm{M}^{+}\right)$.

Acetoxymethyldiphenylsilan (3c): Ausb. $1.6 \mathrm{~g}(80 \%)$, Sdp. $112^{\circ} \mathrm{C} / 0.1$ Torr (Lit. ${ }^{14)} \mathrm{Sdp}$. $132-135^{\circ} \mathrm{C} / 2$ Torr), $n_{\mathrm{D}}^{20}=1.557$ (Lit. $\left.{ }^{14)} n_{\mathrm{D}}^{20}=1.5530\right)$. - IR (Film): $1720 \mathrm{~cm}^{-1}(\mathrm{C}=\mathrm{O})$. ${ }^{1} \mathrm{H}-\mathrm{NMR}\left(\mathrm{CCl}_{4}\right): \delta=0.80\left(\mathrm{~s}, 3 \mathrm{H} ; \mathrm{SiCH}_{3}\right), 2.05\left(\mathrm{~s}, 3 \mathrm{H} ; \mathrm{CCH}_{3}\right), 7.2-7.7\left(\mathrm{~m}, 10 \mathrm{H} ; \mathrm{SiC}_{6} \mathrm{H}_{5}\right)$. MS: $m / e=256\left(<1 \%, \mathrm{M}^{+}\right)$.

Benzoyloxytrimethylsilan (3d): Ausb. $1.6 \mathrm{~g}(80 \%)$, Sdp. $43^{\circ} \mathrm{C} / 0.01$ Torr (Lit. ${ }^{15)} 220^{\circ} \mathrm{C}$ ), $n_{\mathrm{D}}^{20}=1.488\left(\right.$ Lit. $\left.{ }^{15)} n_{\mathrm{D}}^{20}=1.4858\right)$. IR (Film): $1700 \mathrm{~cm}^{-1}(\mathrm{C}=0) .-{ }^{1} \mathrm{H}-\mathrm{NMR}\left(\mathrm{CCl}_{4}\right): \delta=$ $0.31\left(\mathrm{~s}, 9 \mathrm{H} ; \mathrm{SiCH}_{3}\right), 7.1-7.3$ und 8.0-8.2 (m, 5H; $\left.\mathrm{CC}_{6} \mathrm{H}_{5}\right) .-\mathrm{MS}: m / e=194\left(9 \%, \mathrm{M}^{+}\right)$.

Acetoxy([D]methyl)diphenylsilan (3e): Ausb. $1.6 \mathrm{~g} \mathrm{(80 \% ),} \mathrm{Sdp.} 112^{\circ} \mathrm{C} / 0.1$ Torr. $-{ }^{1} \mathrm{H}-\mathrm{NMR}$ $\left(\mathrm{CCl}_{4}\right): \delta=0.78\left(1: 1: 1-\mathrm{t},{ }^{2} J_{\mathrm{HD}}=2.5 \mathrm{~Hz}, 2 \mathrm{H} ; \mathrm{SiCH}_{2} \mathrm{D}\right), 2.05\left(\mathrm{~s}, 3 \mathrm{H} ; \mathrm{CCH}_{3}\right), 7.2-7.7(\mathrm{~m}$, $\left.10 \mathrm{H} ; \mathrm{SiC}_{6} \mathrm{H}_{5}\right) .-{ }^{13} \mathrm{C}-\mathrm{NMR}\left(\mathrm{CDCl}_{3}\right): \delta=-3.0\left(1: 1: 1-\mathrm{t},{ }^{1} J_{\mathrm{CD}}=18.7 \mathrm{~Hz} ; \mathrm{SiCH}_{2} \mathrm{D}\right), 127.8$, 130.2, 133.9 und $134.4\left(\mathrm{SiC}_{6} \mathrm{H}_{5}\right), 171.1(\mathrm{C}=\mathrm{O})$. $-\mathrm{MS}: m / e=257\left(<1 \%, \mathrm{M}^{+}\right)$.

$\left(\left[D_{3}\right]\right.$ Acetoxy)dimethylphenylsilan (3f): Ausb. $1.6 \mathrm{~g}(80 \%)$, Sdp. $67-69^{\circ} \mathrm{C} / 0.05$ Torr. ${ }^{1} \mathrm{H}-\mathrm{NMR}\left(\mathrm{CCl}_{4}\right): \delta=0.53\left(\mathrm{~s}, 6 \mathrm{H} ; \mathrm{SiCH}_{3}\right), 7.2-7.7\left(\mathrm{~m}, 10 \mathrm{H} ; \mathrm{SiC}_{6} \mathrm{H}_{5}\right) .-\mathrm{MS}: m / e=197(3 \%$, $\left.\mathrm{M}^{+}\right)$. 
Kreuzungsexperiment mit $2 \mathrm{~b}$ und $2 \mathrm{e}$ : Ein Gemisch aus $2.91 \mathrm{~g}(15 \mathrm{mmol}) 2 \mathrm{~b}$ und $3.86 \mathrm{~g}$ (15 mmol) $2 \mathrm{e}$ wurde $4 \mathrm{~h}$ unter vermindertem Druck auf $180^{\circ} \mathrm{C}$ gehalten. Durch fraktionierende Vakuumdestillation über eine Spaltrohrkolonne isolierte man zwei Produkte, die sich durch Vergleich $\left({ }^{1} \mathrm{H}\right.$ - und ${ }^{13} \mathrm{C}$-NMR, MS) mit authentischen Proben (s.oben) als $3 \mathrm{~b}$ und $3 \mathrm{e}$ erwiesen.

Kreuzungsexperimente mit $2 \mathrm{c}$ und 2f; a) In Substanz: Ein Gemisch aus $2.56 \mathrm{~g} \mathrm{(10} \mathrm{mmol)} 2 \mathrm{c}$ und $1.97 \mathrm{~g}(10 \mathrm{mmol}) 2 \mathrm{f}$ wurde $4 \mathrm{~h}$ bei 100 Torr auf $160^{\circ} \mathrm{C}$ gehalten. Die Vakuumdestillation ubber eine Spaltrohrkolonne lieferte zwei Fraktionen, die sich durch eine ${ }^{1} \mathrm{H}-\mathrm{NMR}$-spektroskopische Analyse jeweils als ein 1: 1-Gemisch aus $3 \mathrm{~b}$ und $3 \mathrm{f}$ bzw. $3 \mathrm{c}$ und $3 \mathrm{~g}$ erwiesen: Beobachtet wurden die ${ }^{\prime} \mathrm{H}-\mathrm{NMR}$-Spektren $\left(\mathrm{CCl}_{4}\right)$ von $3 \mathrm{~b}$ bzw. $3 \mathrm{c}$, jedoch jeweils nur mit der halben relativen Intensität der $\mathrm{C}-\mathrm{CH}_{3}$-Einheit bei $\delta=2.00(3 \mathrm{~b})$ bzw. $2.05(3 \mathrm{c})$.

b) In benzolischer Lösung: Eine Lösung von $25.6 \mathrm{mg}(0.1 \mathrm{mmol}) 2 \mathrm{c}$ und $19.7 \mathrm{mg}(0.1 \mathrm{mmol}) 2 \mathrm{f}$ in $0.4 \mathrm{ml} \mathrm{C} \mathrm{C}_{6} \mathrm{D}_{6}$ wurde in einem zugeschmolzenen NMR-Röhrchen $4 \mathrm{~h}$ auf $160^{\circ} \mathrm{C}$ erhitzt. Die ${ }^{1} \mathrm{H}$-NMR-Analyse (nach Verdampfen von $\mathrm{C}_{6} \mathrm{D}_{6}$ und Lösen des Rückstandes in $\mathrm{CCl}_{4}$ ) ergab das gleiche Ergebnis wie unter a).

Umsetzung von 3c mit 3f: Eine Mischung von 3c und 3f bzw. eine verdünnte Lösung dieser Substanzen in $\mathrm{CCl}_{4} /\left[\mathrm{D}_{12}\right]$ Cyclohexan $(9: 1)$ ergaben bei Raumtemp. wenige Minuten (ohne Losungsmittel) bzw. einige Tage (mit Lösungsmittel) nach ihrer Herstellung ${ }^{1} \mathrm{H}-\mathrm{NMR}$-Spektren, die ein im Gleichgewicht befindliches Gemisch aus $3 \mathrm{~b}, 3 \mathbf{3}, \mathbf{3 f}$ und $\mathbf{3 g}$ anzeigten.

Kinetische Untersuchungen: $2 \mathrm{a}-\mathrm{e}$ wurden als $0.4-0.5 \mathrm{M}$ benzolische Lösungen - die Verbindungen $2 b$ und $2 c$ daruber hinaus auch pur - unter Stickstoff in NMR-Röhrchen eingeschmolzen. Die einzelnen Proben wurden dann jeweils für 6-9 unterschiedlich lange Zeitrăume innerhalb der ersten 2-3 Halbwertszeiten auf die in Tab. 1 angegebenen Temperaturen erhitzt und nach Abschrecken auf $20^{\circ} \mathrm{C}{ }^{1} \mathrm{H}$-NMR-spektroskopisch untersucht: Die Kinetik der Umlagerung wurde durch Integration der charakteristischen Resonanzsignale der Edukte und Produkte erfaßt. Die so ermittelten Geschwindigkeitskonstanten $k$ (mindestens innerhalb $10 \%$ reproduzierbar) sind in Tab. 1 zusammengestellt.

1) R. Tacke, $H$. Lange und A. Bentlage, Chem. Ber. 115, 3673 (1982).

2) Eine neuere Literaturzusammenfassung uber baseninduzierte 1,2-Hydridverschiebungen vom Silicium zum Kohlenstoff findet sich in: $R$. Tacke, H. Lange und $M$. T. Attar-Bashi, Liebigs Ann. Chem. 1982, 1946.

3) Ein analoger Acetoxy-Austausch zwischen den beiden Isotopomeren $\left[\left(\mathrm{C}_{6} \mathrm{H}_{5}\right)_{2}\right.$ $\left.\left(\mathrm{CD}_{3}\right) \mathrm{C}\right] \mathrm{Si}\left[\mathrm{OC}(\mathrm{O}) \mathrm{CH}_{3}\right]\left(\mathrm{CD}_{3}\right)_{2}$ und $\left[\left(\mathrm{C}_{6} \mathrm{H}_{5}\right)_{2}\left(\mathrm{CH}_{3}\right) \mathrm{C}\right] \mathrm{Si}\left[\mathrm{OC}(\mathrm{O}) \mathrm{CD}_{3}\right]\left(\mathrm{CH}_{3}\right)_{2}$ findet offenbar nicht statt: $N$. Greif, Dissertation, Univ. Marburg 1977; vgl. auch Lit. ${ }^{7}$.

4) M. Kumada, M. Ishikawa und K. Tamao, J. Organomet. Chem. 5, 226 (1966).

5) W. I. Bevan, R. N. Haszeldine, J. Middleton und A. E. Tipping, J. Organomet. Chem. 23, Ci7 (1970).

6) A. R. Bassindale, A. G. Brook, P. F. Jones und J. M. Lennon, Can. J. Chem. 53, 332 (1975).

7) M. T. Reetz und N. Greif, Angew. Chem. 89, 765 (1977); Angew. Chem., Int. Ed. Engl. 16, 712 (1977).

8) D. J. Ager und I. Fleming, J. Chem. Res. (S) 1977, 6.

9) $K$. H. Pannell, J. Organomet. Chem. 21, P 17 (1970).

10) K. H. Pannell und J. R. Rice, J. Organomet. Chem. 78, C35 (1974).

11) $R$. Tacke und $H$. Lange, unveröffentlichte Ergebnisse.

12) $R$. Tacke, $A$. Bentlage, $M$. Link und $H$. Linoh, Publikation in Vorbereitung; $R$. Tacke, M. T. Attar-Bashi, A. Bentlage und $H$. Lange, $6^{\text {th }}$ International Symposium on Organosilicon Chemistry, Budapest 1981, Abstracts of Papers, S. 204.

13) $L$. W. Breed und $R$. L. Elliott, J. Organomet. Chem. 11, 447 (1968).

14) K. A. Andrianov und N. V. Delazari, Dokl. Akad. Nauk SSR 122, 393 (1958) [Chem. Abstr. $53,2133(1959)]$.

15) E. W. Abel, J. Chem. Soc. 1961, 4933.

16) G. Fritz und M. Hähnke, Z. Anorg. Allg. Chem. 390, 157 (1972). 